\title{
Content based Image Processing using Relevance Feedback with Null Space LDA (NLDA)
}

\author{
Rajivegandhi.C \\ Department of Computer Science and Engg \\ Manonmaniam Sundaranar University \\ Tirunelveli, Tamil Nadu \\ India
}

\author{
V. Murugesh, PhD. \\ Associate Professor \\ Department of Computer Science \\ College of Computer Science \\ King Khalid University \\ Kingdom of Saudhi Arabia
}

\begin{abstract}
The biggest problem in the research of Content Based Image Retrieval (CBIR) is bridge the gap between low-level features and high-level semantics., Still many shortcomings for image retrieval system only with the low level visual features due to the semantic space. It is better for the relevance feedback based on the user involvement in image retrieval system. By using the help of user's feedback, the resultant high-level semantic will be obtained. Relevance feedback is a technique for incorporating semantic information in image retrieval. This paper illustrates a development upon a relevance feedback approach that utilizes semantic grouping and clustering technique to close the distance between low-level features and high-level semantics. Distinctively, the past system is improved by incorporating the images in the same group as the query image in the collection of retrieved images. Shared the retrieval results with relevance feedback technology, image feature dimensional reduction was prepared using the Clustering concepts. The given system reduces semantic gap and the storage of image signatures, and also improves the retrieval efficiency and performance. The result shows the efficiency of our proposed system.
\end{abstract}

\section{Keywords}

Content Based Image Processing, Semantic Grouping, Null Space LDA.

\section{INTRODUCTION}

Content-based image retrieval is a expertise that helps to organize picture library by their visual content. With the rapid growth of computing, and digital image acquirement devices available, how to effectively retrieval images in a records is still an challenging issue [1]. Recent years have seen a huge increase in the amount of visual information available to personal computer users. Like other information, it is logical and preferable to systematize this information so that it is categorized or sorted for personal or commercial use. One example of such group is the ability to find images in a data set that are similar to query. At the same time it is technically possible to have users contribute tag information to images, as the number of images in the database increases, it becomes much more of a trouble on the users. At last, it makes more sense to have an automated system that can find and return related images based on the content of a query image, release users to do other things.
Low-level features have been planned as a means of determining the content of images. These include descriptors such as color, shape, and texture [2, 3]. In practice, low-level features only do not supply enough information to depict the high-level semantic concepts of an image. This is in part caused by how differently a user and a computer perceive an image. For example, a user could more easily describe the structures, animals, or atmosphere of an image rather than color, shape, or texture.

Varieties of methods have been utilized to bridge the semantic gap. The more popular method is relevance feedback. Relevance feedback involves receiving feedback from the client as to whether an image returned by the system is relevant to the query. Then it's fed into an algorithm that utilizes the user information to improve later retrieval results. The relevance feedback technique discussed in this paper uses a semantic grouping algorithm to group related images in semantic groups and apply a method called Linear Discriminant Analysis (LDA) to improve retrievals. Semantic grouping uses user feedback to position relevant images together in groups. The LDA technique maximizes the ratio of between-group variance to within-group variance. In this approach, LDA is used to modify the descriptor matrix of the data set so that semantically similar images have slighter Euclidean distances than semantically dissimilar images.Zhou and $\mathrm{Yu}$ Bing et al. demonstrated a system with LDA for face retrieval $[6,7]$. Here, we propose to use information gathered from semantic groups to facilitate retrieval results.

\section{SEMANTIC RELEVANCE FEEDBACK}

In content-based image retrieval, it is difficult for a system to accurately show the search order submitted by users using the signatures of the image. The user's requests will not be preceded successfully, so, we need the help of the relevance feedback techniques. By doing the repeated interaction between the system and users, according to the users' feedback on the estimation of the relevance of the current search results, the searching samples and variety of parameters are adjusted to the users' search requests [5].

Once the query image is submitted to the system, the system returns a specified number of images to the user. The user then marks each returned image as relevant or irrelevant based on his or her semantic perceptive of the match between the query image and returned image. It is assumed that all images in the entire data set are represented in terms of a fixed set of low level features. These low-level features are stored in a vector of real values for each image. The relevance feedback process occurs in the following steps [4]. 
1. Acquire the low-level feature vector for the query image.

2. Calculate the Euclidean distance between the query image feature vector and the rest of the image feature vectors in the data set.

3. If the query image is not already assigned to a semantic group, take the top $\mathrm{k}$ smallest distances and use them as the candidates for the current retrieval. If the query image is already in a group, include the rest of the images in the group in the candidates. Then use as many of the left over smallest distance images until there are $\mathrm{k}$ candidates.

\section{SEMANTIC GROUPING}

Whenever the candidates for the current retrieval are generated, the user can mark each one as relevant (Positive) or irrelevant (Negative). This is called the user's relevance feedback. Then the candidates are assigned to groups in the history through the semantic grouping. The algorithm can be interpreted as events that occur in four possible cases [4].

1. If (any of the negative images has not been assigned to a group), Then assign it to a new group.

2. If (all of the positive images have not been assigned to any groups), Then add all of the positives together to a new semantic group.

3. If (some of the positive images are assigned to some of the groups, and no negative images are in those groups), Then assign all of the positives and the images in their groups to a new semantic group.

4. If (some of the positive images are assigned to some of the groups, and some negative images are in those groups), Then assign all of the positives to a new semantic group, and remove the positives from the groups containing negatives.

\section{MULTI-CLASS LDA}

The system can start generating a new distance metric via LDA once grouping information has been obtained. Basically there are two approaches to LDA: a two-class approach and a multi-class approach. In the two-class approach, all positive images are considered as one class, and all negative images are considered as another class. The aim is to find a distance metric which provides good separation between the two classes. In the multi-class approach, all negative images are considered a class by itself. As in the two-class approach, LDA will effort to maximize the distance between all of the classes. The downside to this approach is that some negative images may actually belong to the same semantic grouping; however, they are grouped into the same semantic group based on the user's relevance feedback record, so allowing them as different classes reduces the effectiveness of LDA.

\section{NULL SPACE LDA (NLDA)}

The null space LDA (NLDA) was proposed to defeat the singularity problem, where the between-class distance is maximized in the null space of the within-class scatter matrix. The singularity problem is thus avoided completely. The efficiency of the algorithm can be enhanced by removing the null space of the total scatter matrix. It is based on the observation that the null space of the total scatter matrix is the intersection of the null spaces of the between-class and within-class scatter matrices. The within scatter matrix $S_{W}$, the between scatter matrix $S_{B}$, and, the total scatter matrix $S_{T}$ are defined as

$$
\begin{aligned}
& S_{w}=\sum_{j=1}^{c} \sum_{i=1}^{N}\left(A_{i}^{j}-\mu_{j}\right)^{T}\left(A_{i}^{j}-\mu_{j}\right) \\
& S_{B}=\sum_{j=1}^{c} N\left(\mu_{j}-\mu\right)^{T}\left(\mu_{j}-\mu\right) \\
& S_{T}=\sum_{j=1}^{c} \sum_{i=1}^{N}\left(A_{i}^{j}-\mu\right)^{T}\left(A_{i}^{j}-\mu\right)=S_{W}+S_{B}
\end{aligned}
$$

Where, $\mu$ is the mean of all samples, and $\mu_{\mathrm{j}}$ is the mean of $\mathrm{N}$ samples in the $\mathrm{j}^{\text {th }}$ class.

The algorithm for Null space is

1. Find the eigenvectors factor $\mathrm{S}_{\mathrm{W}}=\tilde{N} \wedge \tilde{N}^{\prime}$

2. Remove the eigenvectors corresponding to the positive eigenvalues in $\Lambda$. The remaining eigenvectors span the null-space of $S_{W}$. The matrix of remaining eigenvalues is denoted by $\mathrm{N}$. It satisfies $\left\|H^{\prime} S_{W} H\right\|=$ Null.

3. Then the between-class scatter into the within-class scatter: $\tilde{S}_{B}=H^{\prime} S_{B} H$.

4. Find the eigenvector factor $\widetilde{S}_{B}=\tilde{V} \sum \tilde{V}$

5. Choose the k eigenvectors in $\tilde{V}$ corresponding to the largest eigenvalues in $\Sigma$. And their matrix is $\mathrm{V}$. Finally set $\mathrm{W}=\mathrm{NV}$.

\section{EXPERIMENTAL PROCESS}

The experimental parameters in the original paper were used for this project. The data set consisted of 2000 images. For each image, two retrievals were performed for each session, and each retrieval returned 30 images. Another experimental trial was performed in which there were three retrievals per session. The experimental process is described below [4].

1. A query image is arbitrarily chosen from the database

2. If it is the first retrieval of the session, the original distance metric is used. If it is the second or later retrieval, the distance metric computed in the earlier retrieval is used.

3. With the help of distance metric, the 30 smallest distance images are returned to the user. 
4. The user informs the system which images are positive or negative.
6. With the help of updated history, the system computes a new distance metric using LDA.

5. Then the system will update the semantic group history.

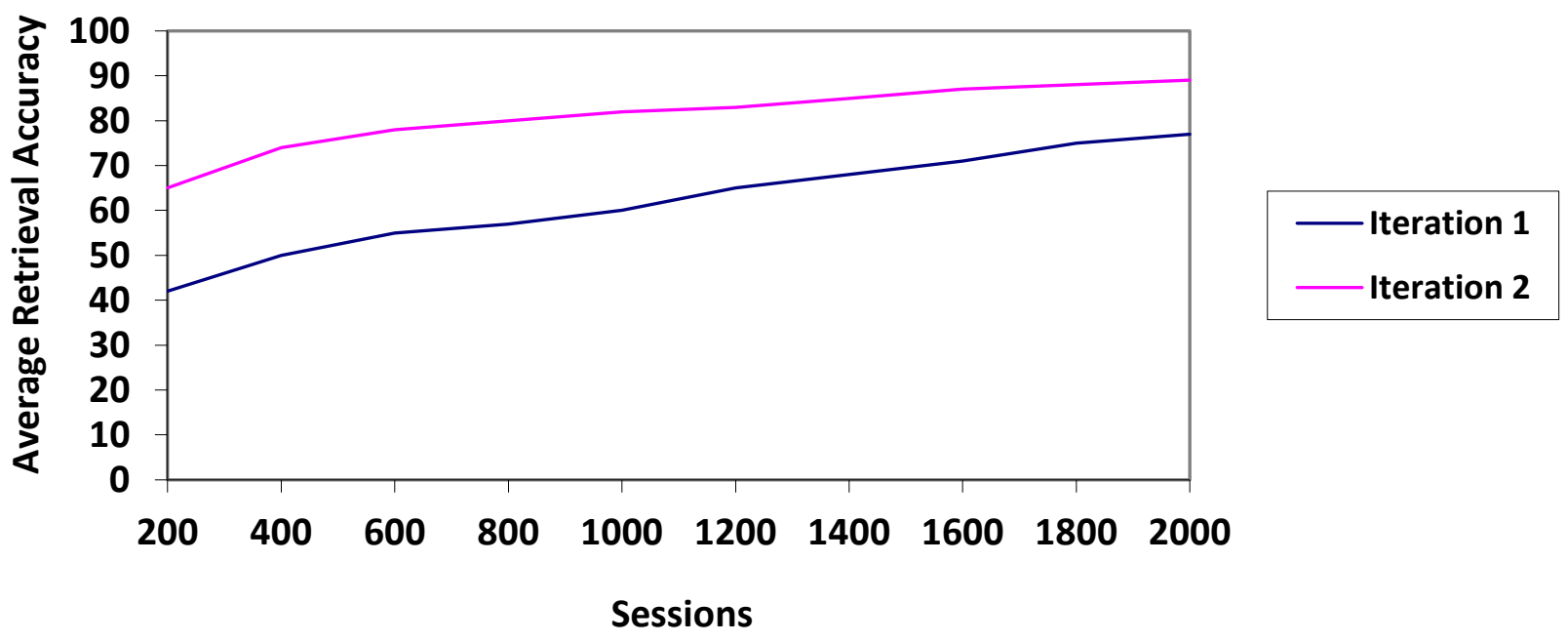

Fig 1: Average Retrieval accuracy for two retrievals

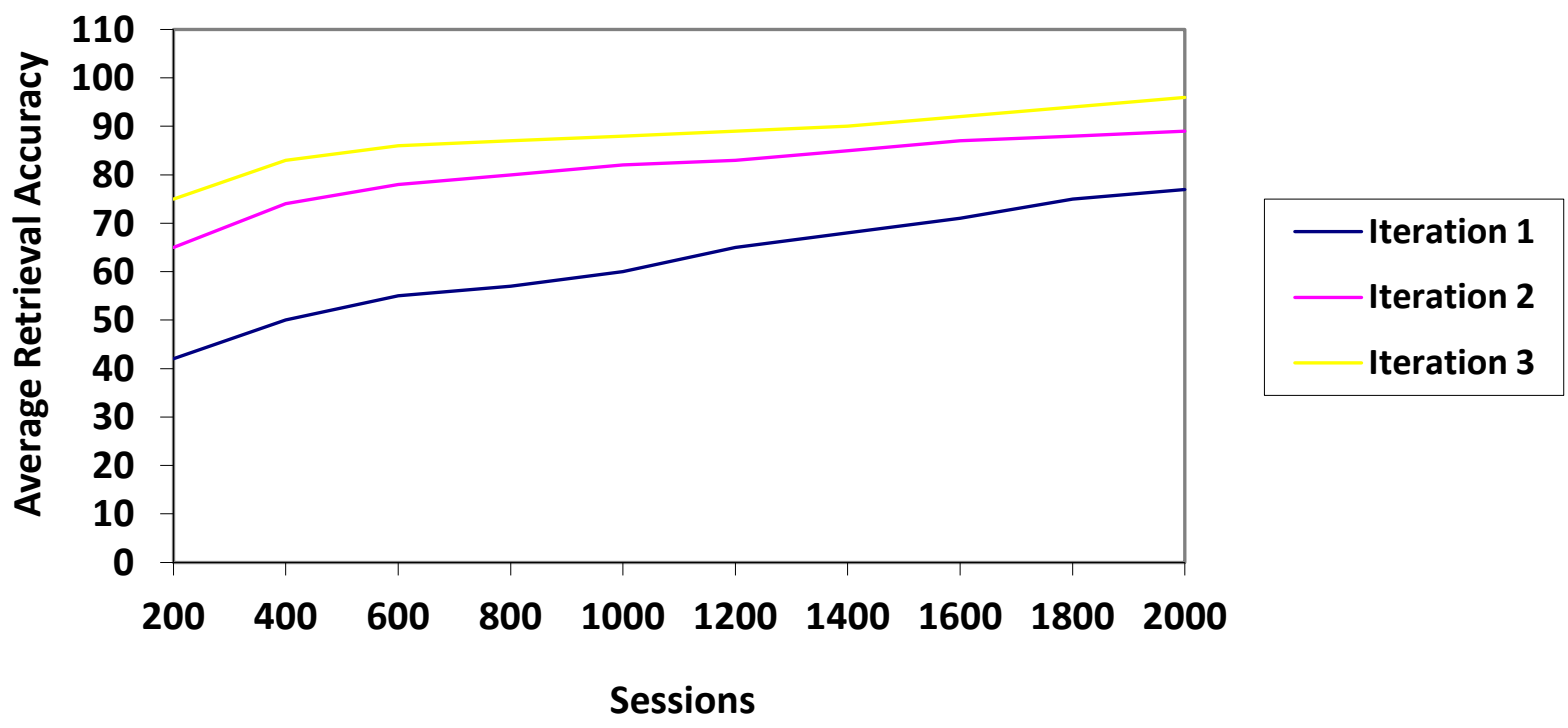

Fig 1: Average Retrieval accuracy for three retrievals

\section{CONCLUSIONS AND FUTURE WORK}

The proposed approach has high performing retrievals after the first retrieval each session. In the original approach, the difference between the first and second retrieval was minor. In our future work, the focus should be on escalating the accuracy of the first retrieval. Also we are planning to implement our image retrieval system using color and texture features of regions $[8,9]$. In addition the performance for large databases remains to be verified. And we will extract the visual features effectively and measure regional similarity to increase the performance of the system.

\section{REFERENCES}

[1] R. Datta, D. Joshi, J. Li, and J. Z. WANG, "Image retrieval: Ideas, influences, and trends of the new age," ACM Computing Surveys, vol. 40, no.2, 2008, pp.1-60 . 
[2] Smith, J. R. and Chang S-F. Transform features for texture classi ${ }^{-}$cation and discrimination in large image databases. In Proceedings of IEEE Intl. Conf. on Image Processing, 1994

[3] Stricker, M. and Orengo, M. Similarity of color images. In Proceedings of SPIE, 1995

[4] T. Yoshizawa and H. Schweitzer. Long-term learning of semantic grouping from relevance- Information Retrival, pages 165 \{172, New York, NY, USA, 2004. ACM.

[5] Zhang Jing, Shen Lan-sun, David Dagan Feng. "A survey of image retrieval based on visual perception", J. ACTA ELECTRONICA SINICA, vol. 36, no. 3, pp. 494-499, 2008
[6] Zhou, X. S. and Huang, T. S. Edge-based structural feature for content-base image retrieval. Pattern Recognition Letters, Special issue on Image and Video Indexing, 2000.

[7] Yu Bing, Jin Lianfu, Chen Ping. A new lda-based method for face recognition. Pattern Recognition, 2002.

[8] Y. Chen and J. Z. Wang, "A region-based fuzzy feature matching approach to content-based image retrieval," IEEE Trans. Pattern Anal.Mach. Intell., vol.24, no.9, 2002, pp.1252-1267.

[9] Y. Chen and J. Z. Wang, "Image categorization by learning and reasoning with regions," Journal of Machine Learning Research, vol.5,2004, pp.913-939. 